\title{
Associations between interleukin-1 gene polymorphisms and sepsis risk: a meta-analysis
}

\author{
An-qiang Zhang, Wei Pan, Jun-wei Gao, Cai-li Yue, Ling Zeng, Wei Gu and Jian-xin Jiang*
}

\begin{abstract}
Background: Previous epidemiological studies have presented conflicting evidence regarding associations between interleukin-1 (IL-1) polymorphisms and sepsis susceptibility. We have performed a meta-analysis to evaluate possible associations between IL-1 polymorphisms and sepsis risk.

Methods: Eligible literature was retrieved from PubMed, Embase and Web of Knowledge databases until Jun 15, 2013. The pooled odds ratio (OR) and $95 \%$ confidence interval (Cl) were calculated using random-effects model in the overall and subgroup analysis based on ethnicity, sepsis severity and quality score.

Results: Eighteen studies addressing five IL-1 polymorphisms were included in this meta-analysis. For IL-1A-889 (rs1800587) polymorphism, significant association was observed in overall comparison for allelic effect ( $\mathrm{OR}=1.47$, $95 \% \mathrm{Cl}=1.01-2.13, \mathrm{P}=0.04$ ). There were no significant associations between either IL-1B-511 (rs 16944) or IL-1B-31 (rs1143627) and sepsis susceptibility in overall or subgroup analyses. For IL-1B +3594 (rs143634) polymorphism, genotype $\Pi$ decreased sepsis risk in overall analysis $(\mathrm{OR}=0.59,95 \% \mathrm{Cl}=0.36-0.97, \mathrm{P}=0.04)$, as well as in Caucasian $(\mathrm{OR}=0.57,95 \% \mathrm{Cl}=0.34-0.95, \mathrm{P}=0.03)$ and sepsis $(\mathrm{OR}=0.55,95 \% \mathrm{Cl}=0.31-0.97, \mathrm{P}=0.04)$ subgroup analysis. For IL-1RN VNTR polymorphism, significant association was observed in overall comparison for allelic effect (OR $=1.40$, $95 \% \mathrm{Cl}=1.01-1.95, \mathrm{P}=0.04)$. Furthermore, the effect sizes of IL-1RN VNTR on sepsis risk increased with disease severity (septic shock $O R>$ severe sepsis $O R>$ sepsis $O R$ ).

Conclusions: Our meta-analysis indicated that IL-1A-889, IL-1B + 3954 and IL-1RN VNTR might be associated with sepsis susceptibility. However, further studies with larger sample sizes and from homogenous populations would be necessary to validate these findings.
\end{abstract}

Keywords: Sepsis, IL-1, Polymorphism, Meta-analysis

\section{Background}

Sepsis is a complex clinical syndrome that results from a systemic inflammatory response to bacteria and/or bacterial products [1]. Though there have been many advances in the development of antibiotics and supportive care, sepsis remains a serious and deadly problem with high mortality rates worldwide [2]. Therefore, predictive markers to identify high-risk patients are urgently needed for early detection and preventive care of sepsis. Cytokines play vital roles in the regulation of host immune response, and altered expression of cytokines is proven to be involved the development of sepsis [3]. Previous research suggests that the variations in the genes encoding cytokines are also

\footnotetext{
*Correspondence: hellojjx@126.com

State Key Laboratory of Trauma, Burns and Combined Injury, Institute of

Surgery Research, Daping Hospital, Third Military Medical University,

Chongqing 400042, China
}

involved in the inflammatory responses and are responsible for inter-individual differences in susceptibility to sepsis and in its severity [4]. Delineating the variations in cytokine genes, and associated differences in response to sepsis might contribute to the development of new genetically tailored diagnostic and therapeutic interventions that may improve outcome in sepsis patients.

Interleukin-1 (IL-1) family is a critical mediator of immune response to sepsis with two agonists (IL- $1 \alpha$ and IL-1 $\beta$ ) and one antagonist (IL-1 receptor antagonist: IL1ra) [5-7]. Excessive IL-1 production is directly linked to the development of shock, multi-organ system failure, and death in patients and animals with sepsis, systemic inflammatory response syndrome, and septic shock [7]. Clinical trials with recombinant IL-1ra in the treatment of sepsis showed the highest mortality rate in those

\section{Biomed Central}

(c) 2014 Zhang et al.; licensee BioMed Central Ltd. This is an open access article distributed under the terms of the Creative Commons Attribution License (http://creativecommons.org/licenses/by/2.0), which permits unrestricted use, distribution, and reproduction in any medium, provided the original work is properly cited. 
patients treated with the largest dose of IL-1ra, suggesting that overproduction of either pro-inflammatory mediators (IL-1 $\alpha$ and IL-1 $\beta$ ) or anti-inflammatory cytokines (IL-1ra) might lead to organ dysfunction and even death [8].

$I L-1 A, I L-1 B$ and $I L-1 R N$ genes (encoding IL- $1 \alpha, I L-1 \beta$, and IL-1ra, respectively) are located next to each other within the cluster of human major histocompatibility complex in the q13-21 area of human chromosome 2 [9]. Five SNPs in $I L-1$ genes have been most frequently studied in relation to sepsis risk: one SNP at promoter position -889 in the $I L-1 A$ gene, two SNPs at promoter position -511 and -31 and one SNP in exon 5 at position +3954 of the $I L-1 B$ gene and a variable number of tandem repeats (VNTR) of 86-bp sequence in intron 2 of $I L-1 R N$ gene, 5 alleles of which have been reported ( 1 to 5 ) corresponding to $4,2,5,3$ and 6 copies of 86-bp sequence, respectively. Thus, the $I L-1 R N$ alleles were further divided into two categories: long genotype (L: including alleles $1,3,4$, and 5) and short genotype (2: allele 2 only). The genotypes were classified as $\mathrm{L} / \mathrm{L}, \mathrm{L} / 2$, and $2 / 2$ [10].

Recently, a number of studies regarding the associations between $I L-1$ polymorphisms and sepsis risk have been published [11-27]. However, the results reported from these studies are inconsistent and inconclusive. We performed a meta-analysis to further investigate the associations between $I L-1$ polymorphisms and sepsis risk, which may help us to better clarify the effect of these polymorphisms on sepsis susceptibility.

\section{Methods}

\section{Identification and eligibility of relevant study}

Relevant articles were identified through a literature search using the keywords "IL-1 or interleukin-1 or IL1" and "sepsis or severe sepsis or septic shock" and "polymorphism or genetic variant or mutation" in Pubmed, Embase, and Web of Knowledge databases until Jun 15, 2013. All searched articles were retrieved and their references were checked for other relevant publications.

The inclusion criteria were: (a) studies evaluating the association between $I L-1$ polymorphisms and sepsis risk, (b) case-control study or cohort design, (c) sufficient data (genotype distributions of cases and controls), and (d) studies written in English. We excluded reviews, comments and articles from which the necessary data could not be extracted nor obtained after contacting the authors. In cases of overlapping studies, only the study with the largest sample size was included.

To minimize the bias and improve the reliability, two researchers reviewed these articles with the inclusion and exclusion criteria independently and reached a consensus.

\section{Data extraction}

Data extraction was independently performed by two investigators and discrepancies were settled by reaching a consensus. Information such as the first author's name, publication year, country and ethnicity of participants, sepsis severity, genotyping method, genotype number or allele frequencies for cases and controls was collected from each study using a standardized data collection protocol. For studies including subjects of different populations, data were extracted separately.

\section{Qualitative assessment}

Quality assessment was performed with the NewcastleOttawa quality assessment scale (NOS) [28]. A 'star system' has been used to judge data quality based on three broad perspectives: the selection, comparability and outcome of interest. Stars are added up to compare the study quality in a quantitative fashion. The scores ranged from 0 to 9 stars. Studies with scores of 7 stars or greater were considered to be of high quality. Based on these criteria, the content validity was evaluated by JWG and C-LY, and any disagreement was resolved via discussions between and or with the other authors for adjudication.

\section{Statistical analysis}

The associations between $I L-1$ polymorphisms and sepsis risk were estimated by calculating pooled ORs and 95\% CI under the dominant $(B B+A B$ vs. $A A)$, recessive ( $B B$ vs. $\mathrm{AB}+\mathrm{AA}$ ), and allelic ( $\mathrm{B}$ vs. $\mathrm{A})$ genetic models respectively (B represented minor allele, A represented major allele). A random-effects model, using Mantel-Haenszel method, was used to calculate pooled ORs. The significance of pooled ORs was determined with $\mathrm{Z}$ tests $(\mathrm{P}<0.05$ was considered statistically significant). Departure from HardyWeinberg equilibrium (HWE) in controls was tested via a Chi-square test at a significance level of $\mathrm{P}<0.05$. Heterogeneity between studies was assessed using the Cochrane Q test and the $\mathrm{I}^{2}$ statistic [29]. Multivariate meta-regression was performed to explore potential sources of heterogeneity among the following covariates: ethnicity (divided into Asian, Caucasian and others), sepsis severity (divided into sepsis, severe sepsis and septic shock), sources of controls (divided into community- or hospital-based) and sample size using REML method of random-effects when the number of studies was more than or equal to 10 [30]. Subgroup analyses were carried out based on ethnicity, severity of sepsis, and quality score. To assess the stability of the pooled results, sensitivity analysis was performed by sequentially excluding individual studies one by one. The publication bias was examined by funnel plot [31] and Egger's test [32]. To adjust the values for multiple comparisons in subgroup analyses, we used the BenjaminiHochberg $(\mathrm{BH})$ step-up correction method, which control the false discovery rate (FDR) [33]. All statistical tests were performed using Review Manager 5.2 (The Cochrane 
Collaboration, Oxford, UK) and STATA11.0 software (StataCorp LP, College Station, Texas, USA).

\section{Result}

\section{Characteristics of eligible studies}

A total of 429 articles were identified update to Jun 15, 2013 (107 from PubMed, 144 from Embase, and 178 from Web of Knowledge). After scanning the abstracts and checking the full-text articles, seventeen articles met the inclusion criteria. Furthermore, two populations containing Caucasian and Black subjects were included in one article by Johnson et al. [13] which was therefore considered as two independent studies (referred to as Johnson-1 and Johnson-2) in the following data analysis. Thus, 18 studies addressing five polymorphisms in IL-1 gene were included in our meta-analysis (Flow diagram shown in Figure 1).

The characteristics of included studies are listed in Table 1 . Of these 18 studies, eight were conducted in Caucasian, six in Asian, two in Hispanic, one in AfricanAmerican, and one in Jewish/Arabic populations. Except for one study for $I L-1 B+3594$ and another for $I L-1 R N$ $V N T R$, the genotype frequency distributions of others were in agreement with HWE (Table 1). Quality scores for individual studies ranged from 6 to 9 , with $78 \%$ (14 of 18) of the studies being classified as high quality (score $\geq 7$ ) (Additional file 1: Table S1). The classical polymerase chain reaction (PCR) and PCR-restriction fragment length polymorphism (PCR-RFLP) were the most commonly used genotyping method in these studies.

\section{Quantitative data synthesis}

\section{IL-1A-889 (rs1800587) polymorphism}

Only two studies [15,17] with high quality containing 193 cases and 193 controls evaluated the association of the $I L$ 1A-889 polymorphism (rs1800587) with sepsis risk. In overall comparison, a significant association was observed for allelic effect $(\mathrm{OR}=1.47,95 \% \mathrm{CI}=1.01-2.13, \mathrm{P}=0.04)$. There were no statistically significant associations between this polymorphism and sepsis risk under dominant or recessive models (Additional file 2: Figure S1, Table 2).

\section{IL-1B-511 (rs16944) and -31 (rs1143627) polymorphisms}

Seven studies [12-15,17,34] totaling 745 cases and 750 controls were identified in order to investigate the association between $I L-1 B-511$ polymorphism (rs16944) and sepsis risk. Overall, the results showed no associations under any genetic model. Removal of the low score study ([34] with score $=6$ ), did not alter these results. In addition, there was no association in any subgroup analysis, either based on ethnicity or sepsis severity under any genetic model (Additional file 2: Figure S2, Table 2).

For the $I L-1 B-31$ polymorphism (rs1143627), four studies [14-16,22] with high quality totaling 405 cases and 824 controls were identified. Similar to $I L-1 B-511$ polymorphism, there were no significant associations detected between rs1143627 and sepsis risk overall or in any subgroup analysis for any genetic model (Additional file 2: Figure S3, Table 2).

\section{IL-1B + 3594 (rs 143634) polymorphism}

Six studies [13,20,23,24,27] containing 680 cases and 998 controls were identified that evaluated the association between the $I L-1 B+3594$ polymorphism (rs143634) and sepsis risk. In the overall comparison, the $I L-1 B+$ 3594 polymorphism was significantly associated with sepsis risk in the recessive effect $(\mathrm{OR}=0.59,95 \% \mathrm{CI}=$ 0.36-0.97, $\mathrm{P}=0.04, \mathrm{P}_{\mathrm{FDR}}=0.12$ ) (Figure 2, Additional file 2: Figure S4). In the subgroup analyses based on ethnicity and sepsis subtype, the significant results persisted in the Caucasian populations $(\mathrm{OR}=0.57,95 \% \mathrm{CI}=0.34$ $0.95, \mathrm{P}=0.03, \mathrm{P}_{\mathrm{FDR}}=0.09$ ) and in sepsis subgroup patients $\left(\mathrm{OR}=0.55,95 \% \mathrm{CI}=0.31-0.97, \mathrm{P}=0.04, \mathrm{P}_{\mathrm{FDR}}=0.12\right)$. However, the negative results for Asians, severe sepsis, and septic shock group patients might be not reliable because only one study was performed in each subgroup (Table 2). After the exclusion of the study by Fang et al. [27], whose genotypic distribution in controls deviated from HWE $\left(\mathrm{P}_{\mathrm{HWE}}=0.03\right)$, the results did not vary significantly $\left(\mathrm{OR}=0.55,95 \% \mathrm{CI}=0.31-0.97, \mathrm{P}=0.04, \mathrm{P}_{\mathrm{FDR}}=0.12\right.$ for overall studies; $\mathrm{OR}=0.51,95 \% \mathrm{CI}=0.28-0.93, \mathrm{P}=0.03$, $\mathrm{P}_{\mathrm{FDR}}=0.09$ for Caucasian populations). Furthermore, the results of stratified analysis based on quality score were not statistically significant.

\section{IL-1RN VNTR polymorphism}

For the $I L-1 R N$ VNTR polymorphism, ten studies [11,12, 17,19,21,24-27,34] comprised of 701 cases and 1227 controls were identified. However, one study by Bessler et al. [21] that deviated from HWE $\left(\mathrm{P}_{\mathrm{HWE}}=0.002\right)$ was not included into the final meta-analysis. The overall results suggested there was statistically significant association of this polymorphism with sepsis risk under allelic model $\left(\mathrm{OR}=1.40,95 \% \mathrm{CI}=1.01-1.95, \mathrm{P}=0.04, \mathrm{P}_{\mathrm{FDR}}=\right.$ 0.12) (Figure 3, Additional 1: Additional file 2: Figure S5). A similar trend was observed in Asian patients $(\mathrm{OR}=1.69$, 95\% $\mathrm{CI}=1.01-2.84)$ although the results were not statistically significant $\left(\mathrm{P}=0.05, \mathrm{P}_{\mathrm{FDR}}=0.15\right)$. Further stratified analyses based on sepsis severity showed that the effect sizes of IL-1RN VNTR on sepsis risk increased with disease severity (septic shock patients $>$ severe sepsis patients $>$ sepsis patients). These findings needed to be interpreted with caution since only one or two studies were included under the three genetic models. Furthermore, stratified analysis based on quality score indicated that there was not significant association in the high quality studies (Quality score $\geq 7$ ) (Table 2). 


\section{PRISMA 2009 Flow Diagram}
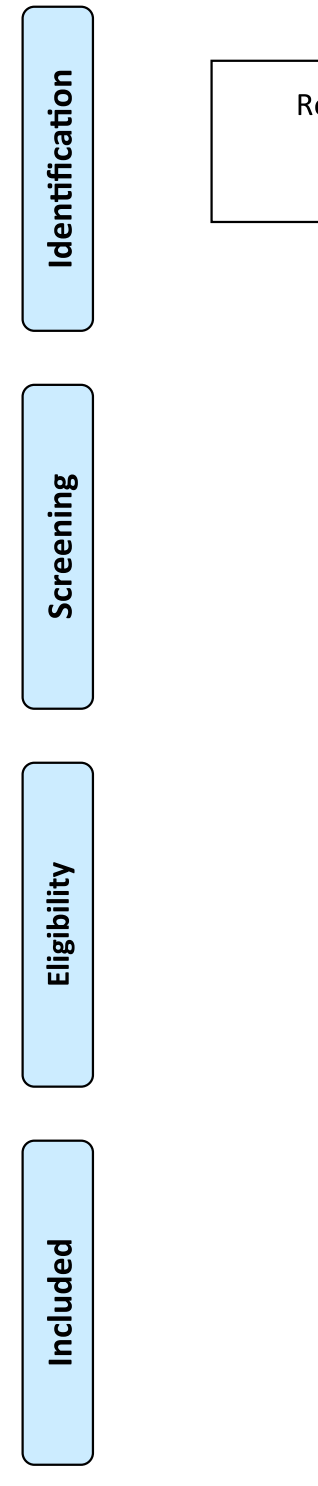
Records identified through database searching $(n=429)$

Additional records identified through reference $(n=0)$

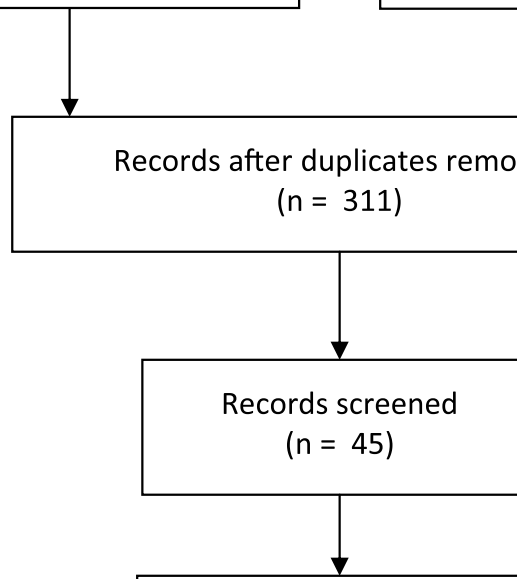

Full-text articles assessed for eligibility $(n=17)$

Records excluded $(n=266)$

Studies included in quantitative synthesis (meta-analysis)*

$$
\begin{gathered}
\text { IL-1A-889 polymorphism }(n=2) \\
\text { IL-1B-511 polymorphism }(n=7) \\
\text { IL-1B-31 polymorphism }(n=4) \\
\text { IL-1B+3594 polymorphism }(n=6) \\
\text { IL-1RN VNTR polymorphism }(n=10)
\end{gathered}
$$

Figure 1 Flow of study identification, inclusion, and exclusion. *represents that certain studies will appear in more than one group when they are divided by polymorphism.

\section{Heterogeneity analysis}

As shown in Table 2 , obvious evidence $\left(\mathrm{P}<0.1\right.$ and $\mathrm{I}^{2}>$ $50 \%)$ for heterogeneity between studies was found for $I L$ $1 B-511$ or $I L-1 R N$ VNTR under all three genetic models, and for $I L-1 B-31$ under dominant and allelic models. No evidence for heterogeneity between studies was found for $I L-1 A-889$ or $I L-1 B+3594$ under any genetic model.
To explore potential sources of between-study heterogeneity in our assessment of IL-1RN VNTR polymorphism and sepsis susceptibility, meta-regression was conducted for all three genetic models. The confounding factors included ethnicity, sepsis severity, sources of controls, and sample size. However, the result did not indicate that any of these potential factors was a major source of 
Table 1 Characteristics of studies included in the meta-analysis

\begin{tabular}{|c|c|c|c|c|c|c|c|c|c|c|c|c|c|c|}
\hline \multirow[t]{2}{*}{ Author/Year } & \multirow[t]{2}{*}{ Country } & \multirow[t]{2}{*}{ Ethnicity } & \multirow{2}{*}{$\begin{array}{c}\text { Sepsis } \\
\text { severity }\end{array}$} & \multirow[t]{2}{*}{ Sources of control } & \multirow{2}{*}{$\begin{array}{l}\text { Genotyping } \\
\text { method }\end{array}$} & \multirow{2}{*}{$\begin{array}{l}\text { Quality } \\
\text { score }\end{array}$} & \multirow{2}{*}{$\begin{array}{c}\text { Case/ } \\
\text { control }\end{array}$} & \multicolumn{3}{|c|}{ Case } & \multicolumn{3}{|c|}{ Control } & \multirow[t]{2}{*}{$P_{\text {HWE }}$} \\
\hline & & & & & & & & $\overline{\mathrm{AA}}$ & $A B$ & $\overline{B B}$ & $\overline{\mathrm{AA}}$ & $A B$ & BB & \\
\hline \multicolumn{15}{|l|}{ IL1a-889C/T } \\
\hline Davis 2010 & America & Hispanic & Sepsis & Community & TaqMan & 7 & $28 / 52$ & 9 & 16 & 3 & 24 & 24 & 4 & 0.55 \\
\hline Gu 2010 & China & Asian & Sepsis & Hospital (trauma) & Probes & 8 & $165 / 141$ & 114 & 42 & 9 & 107 & 31 & 3 & 0.67 \\
\hline \multicolumn{15}{|l|}{ IL-1B-511G/A } \\
\hline Johnson-1 2012 & America & Caucasian & Sepsis & Hospital (noninfected) & Mass-array & 9 & $245 / 263$ & 103 & 114 & 28 & 101 & 120 & 42 & 0.53 \\
\hline Johnson-2 2012 & America & African-American & Sepsis & Hospital (noninfected) & Mass-array & 9 & $93 / 88$ & 28 & 36 & 29 & 26 & 43 & 19 & 0.88 \\
\hline Wan 2012 & China & Asian & Sepsis & Hospital (transplanted) & PCR-RFLP & 7 & $21 / 60$ & 11 & 6 & 4 & 12 & 30 & 18 & 0.94 \\
\hline Shimada 2011 & Japan & Asian & Sepsis & Hospital (critical ill) & Probes & 7 & $123 / 101$ & 33 & 60 & 30 & 35 & 46 & 20 & 0.49 \\
\hline Davis 2010 & America & Hispanic & Sepsis & Community & TaqMan & 7 & $28 / 53$ & 17 & 6 & 5 & 19 & 29 & 5 & 0.20 \\
\hline Gu 2010 & China & Asian & Sepsis & Hospital (trauma) & Probes & 8 & $167 / 140$ & 57 & 83 & 27 & 34 & 61 & 45 & 0.15 \\
\hline Watanabe 2005 & Japan & Asian & Sepsis & Hospital (SIRS) & Probes & 6 & $68 / 45$ & 19 & & $49^{a}$ & 20 & & $25^{\mathrm{a}}$ & 0.86 \\
\hline \multicolumn{15}{|l|}{ IL-1B-31C/T } \\
\hline Shimada 2011 & Japan & Asian & Sepsis & Hospital (critical ill) & Probes & 8 & $122 / 101$ & 36 & 58 & 28 & 34 & 47 & 20 & 0.61 \\
\hline Emonts 2010 & Netherlands & Caucasian & Sepsis & Community & SNaPshot & 7 & $84 / 456$ & 10 & 37 & 37 & 57 & 214 & 185 & 0.69 \\
\hline Gu 2010 & China & Asian & Sepsis & Hospital (trauma) & Probes & 8 & $163 / 144$ & 29 & 106 & 28 & 66 & 60 & 18 & 0.45 \\
\hline Barber 2004 & America & Caucasian & SS & Hospital (burn) & Pyro & 8 & $36 / 123$ & 11 & 13 & 12 & 21 & 54 & 48 & 0.39 \\
\hline \multicolumn{15}{|l|}{$\mathrm{IL}-1 \mathrm{~B}+3594 \mathrm{C} / \mathrm{T}$} \\
\hline Johnson-1 2012 & America & Caucasian & Sepsis & Hospital (noninfected) & Mass-array & 9 & $245 / 263$ & 148 & 89 & 8 & 158 & 88 & 17 & 0.32 \\
\hline Johnson-2 2012 & America & African-American & Sepsis & Hospital (noninfected) & Mass-array & 9 & $93 / 88$ & 61 & 29 & 3 & 65 & 20 & 3 & 0.36 \\
\hline Zhang 2005 & China & Asian & SSH & Hospital (ASP) & PCR-RFLP & 6 & $33 / 76$ & 27 & 6 & 0 & 68 & 8 & 0 & 0.63 \\
\hline Balding 2003 & Ireland & Caucasian & Sepsis & Community & PCR-RFLP & 6 & $183 / 389$ & 113 & 65 & 5 & 240 & 125 & 24 & 0.16 \\
\hline Treszl 2003 & Hungary & Caucasian & Sepsis & Hospital (VLBW) & PCR-RFLP & 6 & $33 / 70$ & 20 & 11 & 2 & 41 & 25 & 4 & 0.94 \\
\hline Fang 1999 & Germany & Caucasian & SS & Community & PCR & 7 & $93 / 112$ & 55 & 31 & 7 & 68 & 33 & 11 & 0.03 \\
\hline \multicolumn{15}{|l|}{ IL-1RN VNTR L/2 } \\
\hline Zapata-Tarres 2013 & Mexico & Hispanic & SSH & Hospital (ALL) & PCR & 8 & $22 / 35$ & 6 & 11 & 5 & 23 & 11 & 1 & 0.82 \\
\hline Wan 2012 & China & Asian & Sepsis & Hospital (transplanted) & PCR-RFLP & 7 & $21 / 60$ & 17 & 4 & 0 & 51 & 9 & 0 & 0.53 \\
\hline Davis 2010 & America & Hispanic & Sepsis & Community & TaqMan & 7 & $28 / 50$ & 12 & 14 & 2 & 27 & 20 & 3 & 0.78 \\
\hline Garcia-Segarra 2007 & Spain & Caucasian & SS and SSH & Community & PCR & 7 & $114 / 80$ & 59 & 52 & 3 & 30 & 41 & 9 & 0.37 \\
\hline Watanabe 2005 & Japan & Asian & Sepsis & Hospital (SIRS) & PCR & 6 & $68 / 45$ & 59 & & $9^{\mathrm{a}}$ & 39 & & $6^{a}$ & 0.54 \\
\hline
\end{tabular}


Table 1 Characteristics of studies included in the meta-analysis (Continued)

\begin{tabular}{ccccccccccccccc}
\hline Bessler 2004 & Israel & Jews/Arabs & Sepsis & Community & PCR & 7 & $34 / 61$ & 18 & 10 & 6 & 33 & 16 & 12 & 0.002 \\
Balding 2003 & Ireland & Caucasian & Sepsis & Community & PCR-RFLP & 6 & $183 / 389$ & 88 & 70 & 25 & 198 & 160 & 31 & 0.87 \\
Arnalich 2002 & Spain & Caucasian & SS & Hospital (CAP) & PCR & 8 & $78 / 186$ & 32 & 33 & 13 & 88 & 81 & 17 & 0.79 \\
Ma 2002 & China & Asian & Sepsis & Community & PCR & 7 & $60 / 60$ & 26 & 27 & 7 & 36 & 21 & 3 & 0.98 \\
Fang 1999 & Germany & Caucasian & SS & Community & PCR & 7 & $93 / 261$ & 37 & 43 & 13 & 152 & 92 & 17 & 0.54
\end{tabular}

SS: severe sepsis; SSH: septic shock; SIRS: systemic inflammatory response syndrome; ASP: acute severe pancreatitis; VLBW: very-low-birth-weight infants; ALL: acute lymphoblastic leukemia; CAP: community-acquired

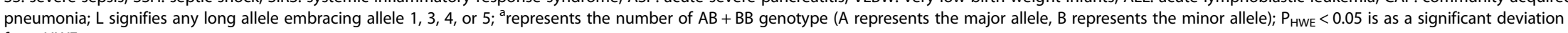
from HWE. 
Table 2 Summary of meta-analysis results

\begin{tabular}{|c|c|c|c|c|c|c|c|c|c|c|c|c|c|c|c|}
\hline & \multicolumn{5}{|c|}{ Dominant model } & \multicolumn{5}{|c|}{ Recessive model } & \multicolumn{5}{|c|}{ Allelic model } \\
\hline & $\mathrm{n}$ & OR $(95 \% \mathrm{Cl})$ & P-value & $P_{\text {het }}$ & $1^{2}$ & $n$ & OR $(95 \% \mathrm{Cl})$ & P-value & $P_{\text {het }}$ & $1^{2}$ & $n$ & OR $(95 \% \mathrm{Cl})$ & P-value & $P_{\text {het }}$ & $1^{2}$ \\
\hline \multicolumn{16}{|l|}{ IL1A-889C/T } \\
\hline Total (sepsis) & 2 & 1.49 (0.95-2.33) & 0.08 & 0.65 & $0 \%$ & 2 & $2.06(0.75-5.67)$ & 0.16 & 0.56 & $0 \%$ & 2 & $1.47(1.01-2.13)$ & 0.04 & 0.98 & $0 \%$ \\
\hline \multicolumn{16}{|l|}{ Ethnicity } \\
\hline Asian & 1 & $1.41(0.85-2.34)$ & 0.19 & & & 1 & $2.65(0.70-10.0)$ & 0.15 & & & 1 & $1.47(0.94-2.30)$ & 0.09 & & \\
\hline Others & 1 & $1.81(0.69-4.74)$ & 0.23 & & & 1 & $1.44(0.30-6.94)$ & 0.65 & & & 1 & $1.46(0.74-2.87)$ & 0.28 & & \\
\hline \multicolumn{16}{|l|}{ IL1B-511G/A } \\
\hline Total (sepsis) & 7 & $0.81(0.53-1.24)$ & 0.33 & $<0.01$ & $68 \%$ & 6 & $0.89(0.53-1.50)$ & 0.66 & $<0.01$ & $67 \%$ & 6 & $0.82(0.60-1.11)$ & 0.20 & $<0.01$ & $69 \%$ \\
\hline \multicolumn{16}{|l|}{ Ethnicity } \\
\hline Asian & 4 & $0.85(0.39-1.87)$ & 0.69 & $<0.01$ & $81 \%$ & 3 & $0.67(0.29-1.55)$ & 0.35 & 0.02 & $73 \%$ & 3 & $0.74(0.38-1.34)$ & 0.29 & $<0.01$ & $84 \%$ \\
\hline Caucasian & 1 & $0.86(0.60-1.23)$ & 0.40 & & & 1 & $0.68(0.41-1.13)$ & 0.14 & & & 1 & $0.84(0.65-1.08)$ & 0.18 & & \\
\hline Others & 2 & $0.63(0.24-1.66)$ & 0.35 & 0.09 & $66 \%$ & 2 & $1.73(0.95-3.14)$ & 0.07 & 0.76 & $0 \%$ & 2 & $0.98(0.58-1.65)$ & 0.93 & 0.18 & $44 \%$ \\
\hline \multicolumn{16}{|l|}{ Quality score } \\
\hline$\geq 7$ & 6 & $0.72(0.48-1.9)$ & 0.12 & 0.02 & $64 \%$ & 6 & $0.89(0.53-1.50)$ & 0.66 & $<0.01$ & $67 \%$ & 6 & $0.82(0.60-1.11)$ & 0.20 & $<0.01$ & $69 \%$ \\
\hline$<7$ & 1 & $2.06(0.94-4.55)$ & 0.07 & & & & & & & & & & & & \\
\hline \multicolumn{16}{|l|}{ IL1B-31C/T } \\
\hline Total (high quality) & 4 & $1.29(0.54-3.06)$ & 0.57 & $<0.01$ & $86 \%$ & 4 & $1.16(0.86-1.56)$ & 0.34 & 0.69 & $0 \%$ & 4 & $1.18(0.78-1.77)$ & 0.43 & $<0.01$ & $78 \%$ \\
\hline \multicolumn{16}{|l|}{ Ethnicity } \\
\hline Asian & 2 & $2.19(0.70-6.90)$ & 0.18 & $<0.01$ & $89 \%$ & 2 & $1.33(0.84-2.09)$ & 0.22 & 0.69 & $0 \%$ & 2 & $1.53(0.91-2.57)$ & 0.11 & 0.04 & $77 \%$ \\
\hline Caucasian & 2 & $0.73(0.33-1.61)$ & 0.43 & 0.15 & $52 \%$ & 2 & $1.04(0.70-1.56)$ & 0.85 & 0.40 & $0 \%$ & 2 & $0.90(0.57-1.43)$ & 0.65 & 0.14 & $55 \%$ \\
\hline \multicolumn{16}{|l|}{ Sepsis severity } \\
\hline Sepsis & 3 & $1.75(0.74-4.11)$ & 0.20 & $<0.01$ & $84 \%$ & 3 & $1.24(0.89-1.72)$ & 0.20 & 0.85 & $0 \%$ & 3 & $1.37(0.93-2.00)$ & 0.11 & 0.03 & $72 \%$ \\
\hline Severe sepsis & 1 & $0.47(0.20-1.10)$ & 0.08 & & & 1 & $0.78(0.36-1.71)$ & 0.54 & & & 1 & $0.68(0.40-1.15)$ & 0.15 & & \\
\hline \multicolumn{16}{|l|}{ Septic shock } \\
\hline \multicolumn{16}{|l|}{ IL1B + 3594C/T } \\
\hline Total & 6 & $1.06(0.86-1.31)$ & 0.57 & 0.79 & $0 \%$ & 5 & $0.59(0.36-0.97)$ & 0.04 & 0.81 & $0 \%$ & 6 & $0.97(0.81-1.15)$ & 0.72 & 0.71 & $0 \%$ \\
\hline \multicolumn{16}{|l|}{ Ethnicity } \\
\hline Asian & 1 & $1.89(0.60-5.96)$ & 0.28 & & & & & & & & 1 & $1.80(0.60-5.41)$ & 0.30 & & \\
\hline Caucasian & 4 & $1.00(0.80-1.25)$ & 0.99 & 0.74 & $0 \%$ & 4 & $0.57(0.34-0.95)$ & 0.03 & 0.74 & $0 \%$ & 4 & $0.92(0.76-1.11)$ & 0.37 & 0.99 & $0 \%$ \\
\hline Others & 1 & $1.48(0.78-2.81)$ & 0.23 & & & 1 & $0.94(0.19-4.81)$ & 0.95 & & & 1 & $1.34(0.77-2.33)$ & 0.31 & & \\
\hline \multicolumn{16}{|l|}{ Sepsis severity } \\
\hline Sepsis & 4 & $1.04(0.83-1.30)$ & 0.75 & 0.71 & $0 \%$ & 4 & $0.55(0.31-0.97)$ & 0.04 & 0.73 & $0 \%$ & 4 & $0.95(0.78-1.15)$ & 0.59 & 0.64 & $0 \%$ \\
\hline
\end{tabular}


Table 2 Summary of meta-analysis results (Continued)

\begin{tabular}{|c|c|c|c|c|c|c|c|c|c|c|c|c|c|c|c|}
\hline Severe sepsis & 1 & $1.07(0.61-1.87)$ & 0.82 & & & 1 & $0.75(0.28-2.01)$ & 0.56 & & & 1 & $0.98(0.62-1.54)$ & 0.93 & & \\
\hline Septic shock & 1 & $1.89(0.60-5.96)$ & 0.28 & & & & & & & & 1 & $1.80(0.60-5.41)$ & 0.30 & & \\
\hline \multicolumn{16}{|l|}{ Quality score } \\
\hline$\geq 7$ & 3 & $1.08(0.82-1.42)$ & 0.57 & 0.55 & $0 \%$ & 3 & $0.63(0.34-1.14)$ & 0.13 & 0.71 & $0 \%$ & 3 & $0.98(0.78-1.23)$ & 0.89 & 0.47 & $0 \%$ \\
\hline$<7$ & 3 & $1.04(0.75-1.43)$ & 0.83 & 0.56 & $0 \%$ & 3 & $0.53(0.23-1.25)$ & 0.15 & 0.37 & $0 \%$ & 3 & $0.95(0.72-1.24)$ & 0.70 & 0.49 & $0 \%$ \\
\hline \multicolumn{16}{|l|}{ IL1RN VNTR L/2 } \\
\hline Total & 9 & $1.39(0.97-1.98)$ & 0.07 & 0.01 & $59 \%$ & 8 & $1.67(0.93-3.02)$ & 0.09 & 0.04 & $54 \%$ & 8 & $1.40(1.01-1.95)$ & 0.04 & $<0.01$ & $72 \%$ \\
\hline \multicolumn{16}{|l|}{ Ethnicity } \\
\hline Asian & 3 & $1.55(0.89-2.68)$ & 0.12 & 0.58 & $0 \%$ & 1 & $2.51(0.62-10.21)$ & 0.20 & & & 2 & $1.69(1.01-2.84)$ & 0.05 & 0.64 & $0 \%$ \\
\hline Caucasian & 4 & $1.16(0.72-1.88)$ & 0.54 & $<0.01$ & $75 \%$ & 4 & $1.39(0.66-2.92)$ & 0.38 & 0.02 & $71 \%$ & 4 & $1.17(0.77-1.78)$ & 0.45 & $<0.01$ & $82 \%$ \\
\hline Others & 2 & $2.68(0.84-8.50)$ & 0.09 & 0.12 & $58 \%$ & 2 & $3.17(0.40-25.32)$ & 0.28 & 0.15 & $52 \%$ & 2 & $2.27(0.78-6.58)$ & 0.13 & 0.05 & $73 \%$ \\
\hline \multicolumn{16}{|l|}{ Sepsis severity } \\
\hline Sepsis & 5 & $1.26(0.95-1.67)$ & 0.11 & 0.69 & $0 \%$ & 4 & $1.85(1.12-3.04)$ & 0.02 & 0.82 & $0 \%$ & 4 & $1.31(1.05-1.64)$ & 0.02 & 0.70 & $0 \%$ \\
\hline Severe sepsis & 2 & $1.67(1.03-2.71)$ & 0.04 & 0.18 & $44 \%$ & 2 & $2.16(1.25-3.72)$ & 0.01 & 0.77 & $0 \%$ & 2 & $1.60(1.18-2.17)$ & $<0.01$ & 0.25 & $24 \%$ \\
\hline Septic shock & 1 & $5.11(1.59-16.46)$ & 0.01 & & & 1 & $10.00(1.08-92.49)$ & 0.04 & & & 1 & $4.00(1.72-9.31)$ & $<0.01$ & & \\
\hline \multicolumn{16}{|l|}{ Quality score } \\
\hline$\geq 7$ & 7 & $1.53(0.95-2.47)$ & 0.08 & $<0.01$ & $67 \%$ & 7 & $1.62(0.72-3.65)$ & 0.24 & 0.02 & $61 \%$ & 7 & $1.46(0.96-2.21)$ & 0.08 & $<0.01$ & $75 \%$ \\
\hline$<7$ & 2 & $1.11(0.79-1.55)$ & 0.55 & 0.84 & $0 \%$ & 1 & $1.83(1.04-3.20)$ & 0.03 & & & 1 & $1.22(0.93-1.60)$ & 0.14 & & \\
\hline
\end{tabular}

Random effects model is used whether or not heterogeneity exists; others contain African-American, Hispanic, and Jews/Arabs; $\mathrm{n}$ : the number of studies involved. OR: odds ratio; Cl: confidence interval; P-value: P value of pooled OR; $\mathrm{P}_{\text {het }}$ : $\mathrm{P}$ of heterogeneity; Statistically significant results $(\mathrm{P}<0.05)$ are highlighted in bold. 


\begin{tabular}{|c|c|c|c|c|c|c|c|c|}
\hline Study or Subgroup & $\begin{array}{l}\text { Case } \\
\text { Events }\end{array}$ & Iotal & $\begin{array}{l}\text { Contrc } \\
\text { Events }\end{array}$ & & Weight & $\begin{array}{l}\text { Odds Ratio } \\
\text { M.H. Randem, } 95 \% \text { cl Year }\end{array}$ & $\begin{array}{r}\text { Odds } \\
M \cdot H, \text { Rand }\end{array}$ & $\begin{array}{l}\text { s Ratio } \\
\text { dem. } 95 \% \mathrm{Cl}\end{array}$ \\
\hline Johnson-1 2012 & 8 & 245 & 17 & 263 & $32.9 \%$ & $0.49[0.21,1.15] 2012$ & & \\
\hline Johnson-2 2012 & 3 & 93 & 3 & 88 & $9.2 \%$ & $0.94[0.19,4.81] 2012$ & & \\
\hline Zhang 2005 & 0 & 33 & 0 & 76 & & Not estimable 2005 & & \\
\hline Treszl 2003 & 2 & 33 & 4 & 70 & $7.9 \%$ & $1.06[0.18,6.13] 2003$ & & \\
\hline Balding 2003 & 5 & 183 & 24 & 389 & $25.3 \%$ & $0.43[0.16,1.14] 2003$ & & \\
\hline Fang 1999 & 7 & 93 & 11 & 112 & $24.8 \%$ & $0.75[0.28,2.01] 1999$ & & \\
\hline Total $(95 \% \mathrm{Cl})$ & & 680 & & 998 & $100.0 \%$ & $0.59[0.36,0.97]$ & & \\
\hline Total events & 25 & & 59 & & & & & \\
\hline \multicolumn{7}{|c|}{$\begin{array}{l}\text { Heterogeneity: } \text { Tau }^{2}=0.00 ; \mathrm{Chi}^{2}=1.58, \mathrm{df}=4(\mathrm{P}=0.81) ; \mathrm{I}^{2}=0 \% \\
\text { Test for overall effect: } Z=2.08(P=0.04)\end{array}$} & 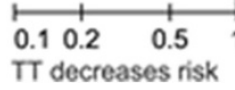 & $\begin{array}{llll}1 & 1 & 1 & \\
\pi \text { increases risk }\end{array}$ \\
\hline \multicolumn{9}{|c|}{$\begin{array}{l}\text { Figure } 2 \text { Forest plot of sepsis susceptibility associated with IL-1B + 3594 polymorphism under the recessive model (TT vs. CT + CC). The } \\
\text { squares and horizontal line represent the individual study-specific OR and } 95 \% \mathrm{Cl} \text {. Area of squares is proportional to the weight of the individual } \\
\text { study to the overall pooled OR. The diamond at the bottom of the graph represents the pooled OR and } 95 \% \mathrm{Cl} \text {. Events': Number of individuals } \\
\text { with TT genotype. OR, odds ratio; Cl, confidence interval. }\end{array}$} \\
\hline
\end{tabular}

heterogeneity (P-value for regression all $>0.05$ ) (Additional file 3: Table S2-S4). Indeed, the heterogeneity might be attributable to other factors, which regrettably remain undefined due to insufficient data. For the other two polymorphisms ( $I L-1 B-511$ and -31), meta-regression was not performed due to the small number of included studies.

\section{Sensitivity analysis}

We removed one study per time from the overall pooled analysis to evaluate the influence of the removed data set on the pooled ORs. The corresponding pooled ORs under any genetic model were not materially altered for $I L-1 A-889, I L-1 B-511$, and -31 , respectively (Additional file 4: Table S5-S7). However, for $I L-1 B+3594$, two studies (Johnson-1 et al. [13] and Balding et al. [24]) altered the corresponding statistical $\mathrm{P}$ value of association under the recessive model (Additional file 4: Table S8b). For $I L$ $1 R N$ VNTR, one study (Garcia-Segarra et al. [19]) was identified as the main cause of heterogeneity. After exclusion of this study, the heterogeneity no longer existed, moreover, positive association was increased under all three genetic models (Additional file 4: Table S9).

\section{Publication bias}

Publication bias was examined by funnel plots and Egger's test under all genetic models. After combining all studies, a little asymmetry was observed for $I L-1 R N V N T R$ polymorphism (Figures 4, 5 and 6), but Egger's test did not show evidence of publication bias (dominant: $\mathrm{P}=0.519$, recessive: $\mathrm{P}=0.724$, allelic: $\mathrm{P}=0.640$ ). For $I L-1 A-889, I L-$ $1 B-511,-31$, and +3594 polymorphisms, publication bias testing was not performed due to the small number of included studies $(\mathrm{n}<9)$.

\section{Discussion}

In this meta-analysis, two polymorphisms $(I L-1 B+3594$ and $I L-1 R N V N T R$ ) were significantly associated with sepsis susceptibility in overall comparison and subgroup

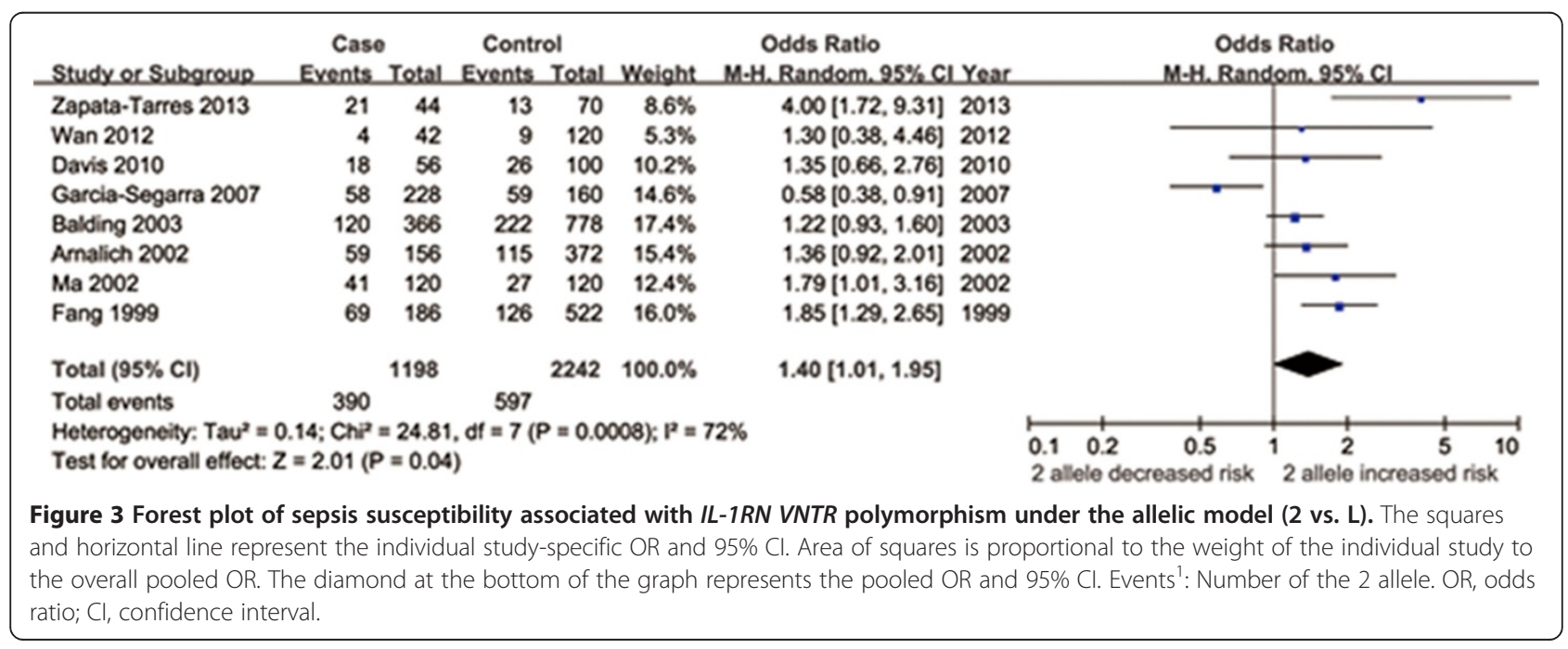




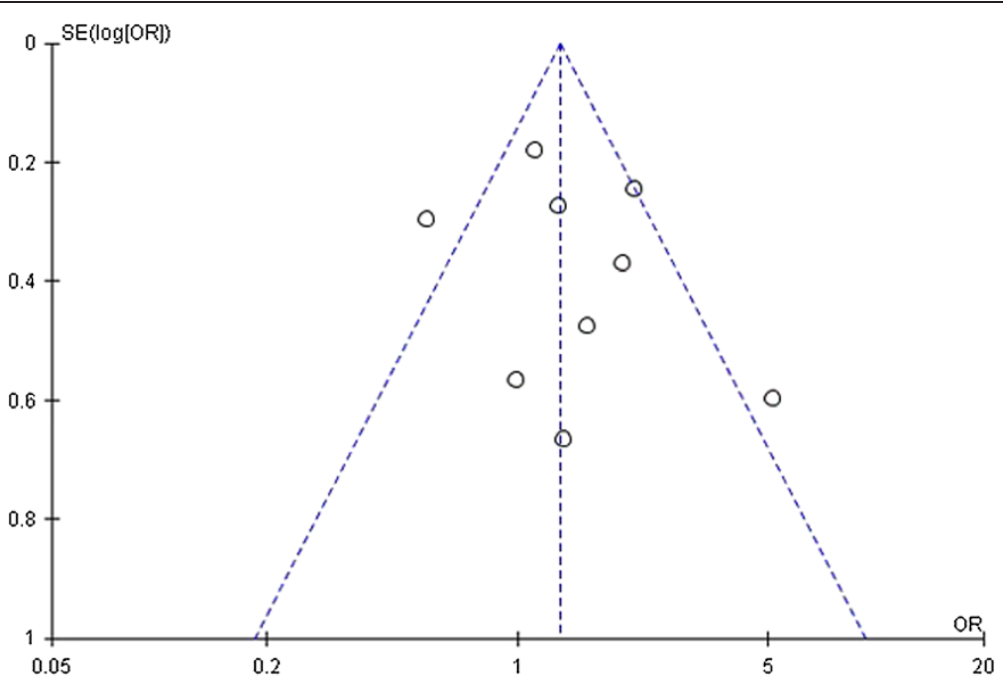

Figure 4 Funnel plot of sepsis susceptibility associated with IL-1RN VNTR under the dominant model $(2 / 2+\mathrm{L} / 2$ vs. L/L). Each circle represents an independent study; for each study the OR was plotted against the standard error of the log of the OR. Center dotted line represents the pooled OR and sloping dotted lines represent the $95 \% \mathrm{Cl}$ of the pooled OR. OR, odds ratio; $\mathrm{SE}$, standard error; $\mathrm{Cl}$, confidence interval.

analyses based on sepsis severity, whereas $I L-1 A-889$ polymorphism influenced sepsis risk only in overall comparison. In contrast, no association was observed between either $I L-1 B-511$ or -31 and sepsis risk in overall comparison or subgroup analyses.

$I L-1 B+3594$ polymorphism is a coding synonymous variant located in exon 5 of $I L-1 B$. The transition from $\mathrm{C}$ to $\mathrm{T}$ does not change amino acid coding but may lead to an inactivation of the original splicing donor site. The alternative splicing results in a premature stop codon or exon skipping and produces a truncated protein that is likely to be rapidly degraded or functionally inactive [35]. The $I L 1 B+3954 T$ allele has been reported to increase the production of IL-1 $\beta$ protein in response to LPS-stimulation [36]. The results of our meta-analysis revealed that individuals with variant genotype (TT) were less susceptible to sepsis than individuals with CC or CT genotypes in overall comparison and Caucasian population, which was inconsistent with higher IL-1 $\beta$ levels associated with increased risk of sepsis. Considering that sepsis is a multifactorial trait and the impact of the inflammatory cytokine on sepsis progress may be modulated by other environmental and

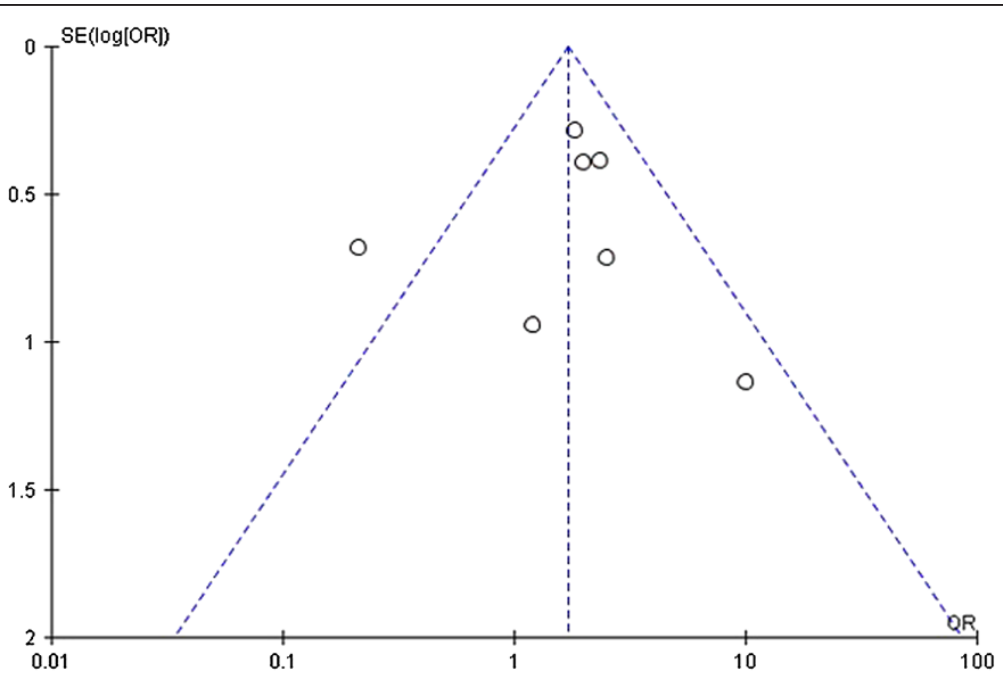

Figure 5 Funnel plot of sepsis susceptibility associated with IL-1RN VNTR under the recessive model ( $2 / 2$ vs. L/2 + L/L). Each circle represents an independent study; for each study the OR was plotted against the standard error of the log of the OR. Center dotted line represents the pooled OR and sloping dotted lines represent the $95 \% \mathrm{Cl}$ of the pooled OR. OR, odds ratio; SE, standard error; Cl, confidence interval. 


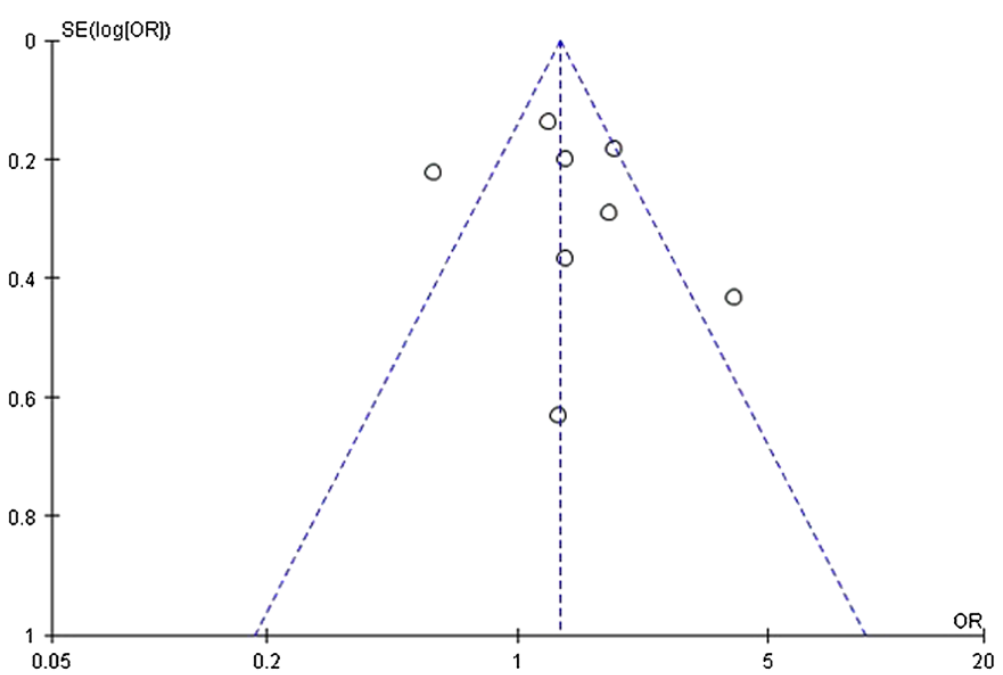

Figure 6 Funnel plot of sepsis susceptibility associated with IL-1RN VNTR under the allelic model (2 vs. L). Each circle represents an independent study; for each study the OR was plotted against the standard error of the log of the OR. Center dotted line represents the pooled OR and sloping dotted lines represent the $95 \% \mathrm{Cl}$ of the pooled OR. OR, odds ratio; SE, standard error; $\mathrm{Cl}$, confidence interval.

genetic factors, more studies should be conducted to clarify the role of $I L-1 B+3594$ polymorphism in the etiology of sepsis.

For the IL-1RN VNTR polymorphism, functional analysis in vitro has shown that the $I L-1 R N-2$ allele correlated with high IL-1ra level and an even more elevated IL-1 $\beta$ level. This resulted in the lowest IL-1ra/IL-1 $\beta$ ratio and was associated with a strengthened and prolonged inflammatory response $[37,38]$. In addition, Arnalich et al. [26] found that $I L-1 R N-2$ allele causes a decreased production of serum IL-1Ra in patients with severe sepsis and ethnically matched healthy controls compared with 1 allele. These findings implied that the $86 \mathrm{bp} \mathrm{VNTR} \mathrm{poly-}$ morphism of the $I L-1 R N$ has potential roles in regulating the immune response and contributed to the pathogenesis of inflammatory diseases. Similarly, our meta-analysis indicated a significant association with risk of sepsis in overall comparison and stratified analysis based on different sepsis severity, which was not observed in ethnicity subgroup analysis. This indicates that the discrepancies between the overall and ethnicity subgroup analyses may be attributed to the diverse genetic backgrounds and environmental factors influencing sepsis risk in different ethnic populations. Further studies with larger samples and from homogeneous populations are warranted to further evaluate the role of the $I L-1 R N$ VNTR polymorphism in sepsis risk in different populations.

IL-1A-889 polymorphism, a C-to-T point mutation in its 5 ' regulatory region, affected over-expression of IL- $1 \alpha$ [35]. In the current meta-analysis, there was significant association with sepsis susceptibility in overall comparison. However, only two studies were included into this meta-analysis with small samples. Thus, this finding needed to be confirmed with larger samples.

The -511 and -31 polymorphisms, which are located at position $511 \mathrm{bp}$ and $31 \mathrm{bp}$ upstream of the transcriptional start site, have been shown to influence the transcriptional activity and expression of the $I L-1 B$ gene. The presence of variant allele of $I L-1 B-511$ markedly increased endotoxin-induced production of IL-1 $\beta$ [36]. Moreover, $I L 1 B-31 C / T$ substitution located in the TATA box motif has been found to markedly affect the binding of several transcription factors and thereby affect the transcriptional activity [39]. In addition, two bi-allelic SNPs $(-511$, and -31$)$ within the human $I L-1 B$ promoter region have been reported to affect LPS-induced IL-1 $\beta$ transcription in vitro and IL- $1 \beta$ plasma levels in healthy adults [40]. Although IL-1 $\beta$ plays an important role in sepsis and IL-1 $\beta$ is frequently over-expressed in sepsis, our meta-analysis indicated no significant association between the $I L-1 B-511$ and -31 polymorphisms and sepsis risk, suggesting that IL-1 $\beta$ expression might influence sepsis progression via mechanisms other than regulation by the two promoter polymorphisms. Several other factors, such as $I L-1 R N$ and NLRP3 may also regulate IL-1 $\beta$ expression $[41,42]$. Further studies are needed to test these hypotheses.

Sepsis is a multifactorial trait and the impact of the inflammatory cytokine on sepsis progress may be modulated by, age, gender and some other environmental factors [43]. Interpretation of the pooled results has been hampered by the fact that several different clinical conditions such as sepsis, severe sepsis, or septic shock have been analyzed together according to the published consensus 
definitions for sepsis [44]. In our stratified analyses, we also examined that whether the effect of polymorphisms differed depending on the way in which sepsis was described (sepsis, severe sepsis, or septic shock). Our results indicated the positive association was observed for $I L-1 B+3594 C / T$ in sepsis group patients, for $I L-1 R N$ $V N T R$ polymorphism in sepsis, severe sepsis, and septic shock group patients, respectively. These findings indicated that the way in which sepsis was described affected the association between $I L-1$ polymorphisms and susceptibility to sepsis. However, what also needs to be pointed out is that these significant associations were derived from only one to two studies and thus the result should be interpreted with caution because of the relatively small sample size.

Heterogeneity is an important problem when interpreting the results of our meta-analysis. In this study, significant heterogeneity was found for the $I L-1 B-511,-31$, and $I L-1 R N$ VNTR polymorphisms. Meta-regression did not seem to reveal the potential sources of heterogeneity with the introduction of covariates containing ethnicity, sepsis severity, sources of controls, sample size, which indicated that the heterogeneity might be attributable to other factors, which regrettably remain undefined due to insufficient data. Moreover, we carried out sensitivity analysis. Removal of each study did not seem to alter the relationships with sepsis risk and heterogeneity for $I L$ $1 B-511$ and -31 , suggesting the reliability of these results. However, removing one study by Garcia-Segarra et al. for $I L-1 R N V N T R$ obviously decreased the heterogeneity and increased positive association under all genetic models, which indicated this study may be the main cause of heterogeneity.

Another important problem is publication bias. Because meta-analyses review quantitative data from numerous studies, the publication bias effect of the literature incorporated in the study can bias the mea-analytic outcome. For IL-1RN VNTR polymorphism, although the Egger's test did not show significant publication bias for sepsis risk, we found the shape of the funnel plot was slightly asymmetrical. Thus, the results should be interpreted cautiously and more studies are still needed to confirm the findings from this meta-analysis.

Some limitations of this meta-analysis should be pointed out. First, the number and sample size of the included studies was limited, some unpublished reports, non-English articles and studies without sufficient information were not included in our meta-analysis, which might bias the pooled results. Second, although the results in stratified analysis were more meaningful, there were a small number of studies in each stratum, thus limiting the interpretation of these analyses. Third, as none of the studies included in this meta-analysis considered the effect of gene-gene/environment involved in the pathogenesis of sepsis, this issue could not be addressed. Fourth, the overall outcome was based on unadjusted data, where as a more precise analysis stratified by variables such as age, sex, type of infection etc. could not be performed due to limitations of the data which also restricted our ability to detect possible sources of heterogeneity. Fifth, stratified analyses based on the different sources of controls were not performed due to small number of studies in each subgroup; the inclusion of studies with varied control populations might increase the probability of typeIerror and bias the pooled results. Finally, as we only focused on the associations between $I L-1$ polymorphisms and sepsis susceptibility in the present study, the significance was limited. To better illuminate the role of IL-1 polymorphisms in outcome of sepsis, it would also be important to perform meta-analyses on the associations between polymorphisms and severity or sepsisrelated mortality in future studies.

\section{Conclusions}

To our knowledge, this is the first study that quantitatively synthesized the association between the $I L-1$ polymorphisms and sepsis. The results demonstrated that the $I L-1 A-889 C / T, I L-1 B-3594 C / T$, and $I L-1 R N$ $V N T R$ polymorphisms had significant associations with the risk of sepsis, although some results were limited by the small number of studies. However, no significant association existed between $I L-1 B-511,-31$ and sepsis risk. Further studies with large samples and homogeneous population are needed to evaluate their associations with sepsis risk.

\section{Additional files}

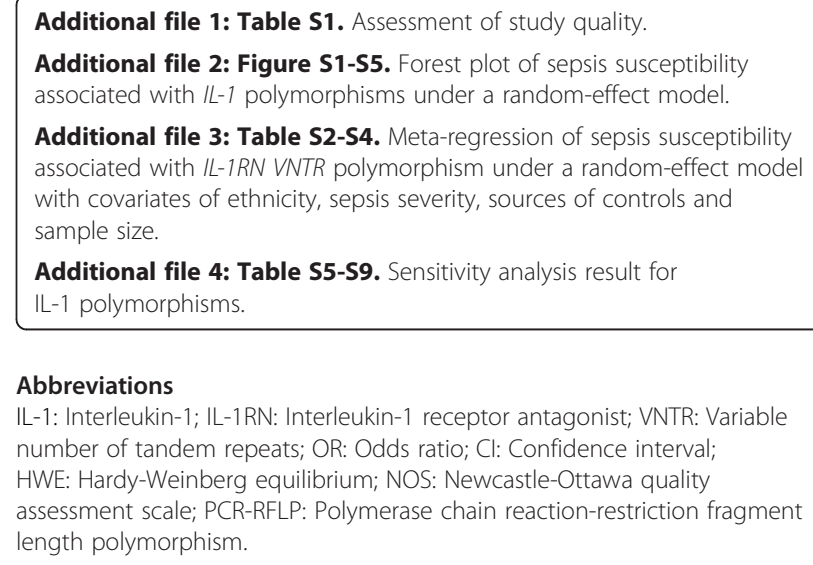

Additional file 4: Table S5-S9. Sensitivity analysis result for IL-1 polymorphisms.

\begin{abstract}
Abbreviations
IL-1: Interleukin-1; IL-1RN: Interleukin-1 receptor antagonist; VNTR: Variable number of tandem repeats; OR: Odds ratio; Cl: Confidence interval; HWE: Hardy-Weinberg equilibrium; NOS: Newcastle-Ottawa quality assessment scale; PCR-RFLP: Polymerase chain reaction-restriction fragment length polymorphism.
\end{abstract}

Competing interests

The authors declare that they have no competing interests.

Authors' contributions

A-QZ and WP was the main researcher for this study and took part in conceptualization, literature review, data extraction, analysis, writing of the manuscript. J-WG, C-LY and LZ were involved in software used, data analysis and final editing. WG did to guide the statistical analysis and manuscript editing. J-XJ planned the study, wrote the protocol and was involved in the 
genetic and clinical aspects of data analyses and revised the manuscript. All authors read and approved the final manuscript.

\section{Acknowledgements}

This work is supported by National Key Technology R\&D Program (2012BAl11B01), Hundreds of outstanding science and technology leading talent research fund in Chongqing City, The Major State Basic Research Development Program of China (2012CB518102), National Natural Science Funds for Distinguished Young Scholar (81201462), and Open fund of State Key Laboratory of Trauma, Burns and Combined Injury, Third Military Medical University (SKLZZ201104). The funders had no role in study design, data collection and analysis, decision to publish, or preparation of the manuscript.

Received: 10 August 2013 Accepted: 12 January 2014

Published: 16 January 2014

\section{References}

1. Rittirsch D, Flierl MA, Ward PA: Harmful molecular mechanisms in sepsis. Nat Rev Immunol 2008, 8(10):776-787.

2. Martin GS, Mannino DM, Eaton S, Moss M: The epidemiology of sepsis in the United States from 1979 through 2000. N Engl J Med 2003, 348(16):1546-1554.

3. Grealy R, White M, Stordeur P, Kelleher D, Doherty DG, McManus R, Ryan T: Characterising cytokine gene expression signatures in patients with severe sepsis. Mediators Inflamm 2013, 2013:164246.

4. Wen $A Q$, Gu W, Zeng L, Zhou J, Jiang DP, Zhang L, Du DY, Hu P, Chen K, Liu Q, Wang ZG, Jiang JX: Clinical relevance of IL-1 beta promoter polymorphisms $(-1470,-511$, and -31$)$ in patients with Chinese major trauma patients. Shock 2010, 33(6):576-582.

5. Kurt AN, Aygun AD, Godekmerdan A, Kurt A, Dogan Y, Yilmaz E: Serum IL-1 beta, IL-6, IL-8, and TNF-alpha levels in early diagnosis and management of neonatal sepsis. Mediators Inflamm 2007, 2007:31397.

6. Fida NM, Al-Mughales J, Farouq M: Interleukin-1alpha, interleukin-6 and tumor necrosis factor-alpha levels in children with sepsis and meningitis. Pediatr Int 2006, 48(2):118-124.

7. Pruitt JH, Copeland EM 3rd, Moldawer LL: Interleukin-1 and interleukin-1 antagonism in sepsis, systemic inflammatory response syndrome, and septic shock. Shock 1995, 3(4):235-251.

8. Opal SM, Fisher CJ Jr, Dhainaut JF, Vincent JL, Brase R, Lowry SF, Sadoff JC, Slotman GJ, Levy H, Balk RA, Shelly MP, Pribble JP, LaBrecque JF, Lookabaugh J, Donovan H, Dubin H, Baughman R, Norman J, DeMaria E, Matzel K, Abraham E, Seneff M: Confirmatory interleukin-1 receptor antagonist trial in severe sepsis: a phase III, randomized, double-blind, placebo-controlled, multicenter trial. The interleukin-1 receptor antagonist sepsis investigator group. Crit Care Med 1997, 25(7):1115-1124.

9. Dinarello CA: Biologic basis for interleukin-1 in disease. Blood 1996, 87(6):2095-2147.

10. Vamvakopoulos JE, Taylor CJ, Morris-Stiff GJ, Green C, Metcalfe S: The interleukin-1 receptor antagonist gene: a single-copy variant of the intron 2 variable number tandem repeat (VNTR) polymorphism. Eur J Immunogenet 2002, 29(4):337-340.

11. Zapata-Tarres M, Arredondo-Garcia JL, Rivera-Luna R, Klunder-Klunder M, Mancilla-Ramirez J, Sanchez-Urbina R, Vazquez-Cruz MY, Juarez-Villegas LE, Palomo-Colli MA: Interleukin-1 receptor antagonist gene polymorphism increases susceptibility to septic shock in children with acute lymphoblastic leukemia. Pediatr Infect Dis J 2013, 32(2):136-139.

12. Wan $\mathrm{QQ}$, Ye QF, Ma Y, Zhou JD: Genetic association of interleukin-1(beta) $(-511 \mathrm{C} / \mathrm{T})$ and its receptor antagonist (86-bpVNTR) gene polymorphism with susceptibility to bacteremia in kidney transplant recipients. Transplant Proc 2012, 44(10):3026-3028.

13. Johnson MD, Plantinga TS, Van De Vosse E, Velez Edwards DR, Smith PB, Alexander BD, Yang JC, Kremer D, Laird GM, Oosting M, Joosten LAB, Van Der Meer JWM, Van Dissel JT, Walsh TJ, Perfect JR, Kullberg BJ, Scott WK, Netea MG: Cytokine gene polymorphisms and the outcome of invasive candidiasis: A prospective cohort study. Clin Infect Dis 2012, 54(4):502-510.

14. Shimada T, Oda S, Sadahiro T, Nakamura M, Hirayama Y, Watanabe E, Abe R, Nakada T-a, Tateishi Y, Otani S, Hirasawa H, Tokuhisa T, Uno H: Outcome prediction in sepsis combined use of genetic polymorphisms - A study in Japanese population. Cytokine 2011, 54(1):79-84.
15. Gu W, Zeng L, Zhou J, Jiang DP, Zhang L, Du DY, Hu P, Chen K, Liu Q, Wang ZG, Jiang JX: Clinical relevance of 13 cytokine gene polymorphisms in Chinese major trauma patients. Intensive Care Med 2010, 36(7):1261-1265.

16. Emonts $\mathrm{M}$, Vermont $\mathrm{CL}$, Houwing-Duistermaat JJ, Haralambous $\mathrm{E}$, Gaast-de Jongh CE, Hazelzet JA, Faust SN, Betts H, Hermans PWM, Levin M, de Groot R, St Mary's Imperial Coll G, Erasmus MCSCsH: Polymorphisms in parp, II1 b, II, I10, C1 inh, Defb1, and Defa4 in meningococcal disease in three populations. Shock 2010, 34(1):17-22.

17. Davis SM, Clark EA, Nelson LT, Silver RM: The association of innate immune response gene polymorphisms and puerperal group a streptococcal sepsis. Am J Obstet Gynecol 2010, 202(3):e301-308.

18. Motoyama S, Miura M, Hinai Y, Maruyama K, Usami S, Nakatsu T, Saito H, Minamiya Y, Murata K, Suzuki T, Ogawa J: Interferon-gamma 874A > T genetic polymorphism is associated with infectious complications following surgery in patients with thoracic esophageal cancer. Surgery 2009, 146(5):931-938.

19. Garcia-Segarra G, Espinosa G, Tassies D, Oriola J, Aibar J, Bove A, Castro P, Reverter J-C, Nicolas J-M: Increased mortality in septic shock with the 4G/4G genotype of plasminogen activator inhibitor 1 in patients of white descent. Intensive Care Med 2007, 33(8):1354-1362.

20. Zhang DL, Zheng HM, Yu BJ, Jiang ZW, Li JS: Association of polymorphisms of IL and CD14 genes with acute severe pancreatitis and septic shock. World J Gastroenterol 2005, 11(28):4409-4413.

21. Bessler H, Osovsky M, Sirota L: Association between IL-1 ra gene polymorphism and premature delivery. Biol Neonate 2004, 85(3):179-183.

22. Barber RC, Aragaki CC, Rivera-Chavez FA, Purdue GF, Hunt JL, Horton JW: TLR4 and TNF-alpha polymorphisms are associated with an increased risk for severe sepsis following burn injury. J Med Genet 2004, 41(11):808-813.

23. Treszl A, Kocsis I, Szathmari M, Schuler A, Heninger E, Tulassay T, Vasarhelyi B: Genetic variants of TNF-[FC12]a, IL-1beta, IL-4 receptor [FC12]a-chain, IL-6 and IL-10 genes are not risk factors for sepsis in low-birth-weight infants. Biol Neonate 2003, 83(4):241-245.

24. Balding J, Healy CM, Livingstone WJ, White B, Mynett-Johnson L, Cafferkey M, Smith OP: Genomic polymorphic profiles in an Irish population with meningococcaemia: is it possible to predict severity and outcome of disease? Genes Immun 2003, 4(8):533-540.

25. Ma P, Chen D, Pan J, Du B: Genomic polymorphism within interleukin-1 family cytokines influences the outcome of septic patients. Crit Care Med 2002, 30(5):1046-1050.

26. Arnalich F, Lopez-Maderuelo D, Codoceo R, Lopez J, Solis-Garrido LM, Capiscol C, Fernandez-Capitan C, Madero R, Montiel C: Interleukin-1 receptor antagonist gene polymorphism and mortality in patients with severe sepsis. Clin Exp Immunol 2002, 127(2):331-336.

27. Fang XM, Schroder S, Hoeft A, Stuber F: Comparison of two polymorphisms of the interleukin-1 gene family: interleukin-1 receptor antagonist polymorphism contributes to susceptibility to severe sepsis. Crit Care Med 1999, 27(7):1330-1334.

28. Wells GA, Shea B, O'Connell D, Peterson J, Welch V, Losos M, Tugwell P: The Newcastle-Ottawa Scale (NOS) for assessing the quality of nonrandomised studies in meta-analyses. Ottawa Health Research Institute; 2011. www.ohri.ca/ programs/dinical_epidemiology/oxford.asp. Accessed 20 October 2011.

29. Higgins JP, Thompson SG, Deeks JJ, Altman DG: Measuring inconsistency in meta-analyses. BMJ 2003, 327(7414):557-560.

30. Schmid CH, Stark PC, Berlin JA, Landais P, Lau J: Meta-regression detected associations between heterogeneous treatment effects and study-level, but not patient-level, factors. J Clin Epidemiol 2004, 57(7):683-697.

31. Sutton AJ, Duval SJ, Tweedie RL, Abrams KR, Jones DR: Empirical assessment of effect of publication bias on meta-analyses. BMJ 2000, 320(7249):1574-1577.

32. Egger M, Davey Smith G, Schneider M, Minder C: Bias in meta-analysis detected by a simple, graphical test. BMJ 1997, 315(7109):629-634.

33. Noble WS: How does multiple testing correction work? Nat Biotechnol 2009, 27(12):1135-1137.

34. Watanabe E, Hirasawa H, Oda S, Shiga H, Matsuda K, Nakamura M, Abe R, Nakada T: Cytokine-related genotypic differences in peak interleukin-6 blood levels of patients with SIRS and septic complications. J Trauma 2005, 59(5):1181-1189. discussion 1189-1190.

35. Shirodaria S, Smith J, McKay IJ, Kennett CN, Hughes FJ: Polymorphisms in the IL-1A gene are correlated with levels of interleukin-1alpha protein in gingival crevicular fluid of teeth with severe periodontal disease. J Dent Res 2000, 79(11):1864-1869. 
36. Pociot F, Molvig J, Wogensen L, Worsaae H, Nerup J: A Taql polymorphism in the human interleukin-1 beta (IL-1 beta) gene correlates with IL-1 beta secretion in vitro. Eur J Clin Invest 1992, 22(6):396-402.

37. Witkin SS, Gerber S, Ledger WJ: Influence of interleukin-1 receptor antagonist gene polymorphism on disease. Clin Infect Dis 2002, 34(2):204-209.

38. Lim WY, Chen Y, Ali SM, Chuah KL, Eng P, Leong SS, Lim E, Lim TK, Ng AW Poh WT, Tee A, Teh M, Salim A, Seow A: Polymorphisms in inflammatory pathway genes, host factors and lung cancer risk in Chinese female never-smokers. Carcinogenesis 2011, 32(4):522-529.

39. Chen H, Wilkins LM, Aziz N, Cannings C, Wyllie DH, Bingle C, Rogus J, Beck JD, Offenbacher S, Cork MJ, Rafie-Kolpin M, Hsieh CM, Kornman KS, Duff GW: Single nucleotide polymorphisms in the human interleukin-1B gene affect transcription according to haplotype context. Hum Mol Genet 2006, 15(4):519-529.

40. Wen AQ, Wang J, Feng K, Zhu PF, Wang ZG, Jiang JX: Effects of haplotypes in the interleukin 1 beta promoter on lipopolysaccharide-induced interleukin 1beta expression. Shock 2006, 26(1):25-30

41. Santtila S, Savinainen K, Hurme M: Presence of the IL-1RA allele 2 (IL1RN*2) is associated with enhanced IL-1 beta production in vitro. Scand I Immunol 1998, 47(3):195-198.

42. Weber A, Wasiliew P, Kracht M: Interleukin-1 beta (IL-1 beta) processing pathway. Sci Signal 2010, 3(105):cm2.

43. Watanabe E, Buchman TG, Hirasawa H, Zehnbauer BA: Association between lymphotoxin-(alpha) (tumor necrosis factor-(beta)) intron polymorphism and predisposition to severe sepsis is modified by gender and age. Crit Care Med 2010, 38(1):181-193.

44. Levy MM, Fink MP, Marshall JC, Abraham E, Angus D, Cook D, Cohen J, Opal SM, Vincent UL, Ramsay G: 2001 SCCM/ESICM/ACCP/ATS/SIS International Sepsis Definitions Conference. Crit Care Med 2003, 31(4):1250-1256.

doi:10.1186/1471-2350-15-8

Cite this article as: Zhang et al: Associations between interleukin-1 gene polymorphisms and sepsis risk: a meta-analysis. BMC Medical Genetics 2014 15:8

\section{Submit your next manuscript to BioMed Central and take full advantage of:}

- Convenient online submission

- Thorough peer review

- No space constraints or color figure charges

- Immediate publication on acceptance

- Inclusion in PubMed, CAS, Scopus and Google Scholar

- Research which is freely available for redistribution 